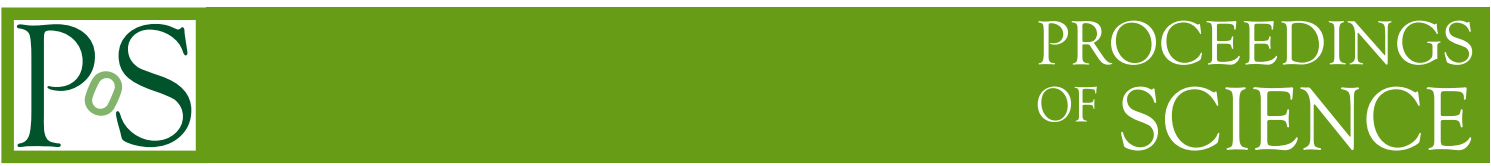

\title{
Long-baseline neutrino oscillation sensitivities with Hyper-Kamiokande
}

\author{
Mark Scott ${ }^{a, *}$ \\ ${ }^{a}$ Department of Physics, Imperial College London, \\ Prince Consort Road, London, United Kingdom
}

E-mail: m.scott09@imperial.ac.uk

The discovery of neutrino oscillations implies that neutrinos are massive particles, which in turn requires new physics beyond the Standard Model. Over the past two decades the study of neutrino oscillations within the PMNS paradigm has produced measurements of all three mixing angles and both mass-splittings. Neutrino oscillation also provides a mechanism to violate the CP symmetry which would be a clue towards understanding the origin of the matter-antimatter asymmetry of the universe. Measuring CP violation in neutrino oscillation is one of the goals of Hyper-Kamiokande. Hyper-Kamiokande is a next-generation water Cherenkov detector that is under construction in Japan. The baseline design has a fiducial volume 8 times the size of the currently-running Super-Kamiokande detector and is instrumented with new photosensors that offer significant improvements in performance. Combined with the upgraded J-PARC neutrino beam, Hyper-Kamiokande will be able to measure neutrino oscillations with an unprecedented statistical precision. This talk presents the sensitivity of Hyper-Kamiokande to CP violation along with the expected precision on the other oscillation parameters and mass hierarchy.

40th International Conference on High Energy physics - ICHEP2020

July 28 - August 6, 2020

Prague, Czech Republic (virtual meeting)

\footnotetext{
${ }^{*}$ Speaker
} 


\section{Introduction}

Hyper-Kamiokande (Hyper-K ) is a large-scale water Cherenkov detector that will study both neutrinos produced by the J-PARC accelerator complex as well as neutrinos from natural sources. The Hyper-K project was approved in Japan earlier this year, construction has started and data taking is expected to begin in 2027.

The detector will be eight times the size of the existing Super-Kamiokande experiment, with a fiducial volume of approximately 188,000 tonnes, and will be sited in the Tochibora mine $295 \mathrm{~km}$ from J-PARC. Combined with an upgrade of the neutrino beam power, Hyper-K will collect 20 times more long-baseline neutrino events than the $\mathrm{T} 2 \mathrm{~K}$ experiment, opening the door to precision measurement of neutrino oscillations.

\section{Open questions in neutrino oscillations}

One of the biggest unanswered questions in physics is the baryon asymmetry of the Universe (BAU). Sakharov's conditions [1] require there to have been a process that violated the ChargeParity (CP) symmetry by a sizeable amount. CP violation has so far only been observed in quark systems, and is too small to meet the requirements of the BAU. Neutrino oscillations provide another mechanism for $\mathrm{CP}$ violation and it has been shown that this could produce the observed baryon asymmetry through a process known as leptogenesis [2].

Assuming that there are three neutrino flavours, the neutrino oscillation probability can be fully specified by six parameters: three mixing angles, $\theta_{i j}$, two mass-squared splittings, $\Delta m_{i j}^{2}$ and a complex phase, $\delta_{\mathrm{CP}}$. For the Hyper-K baseline the probability for a muon neutrino to oscillate to an electron neutrino is given by:

$$
\mathrm{P}\left(v_{\mu} \rightarrow v_{e}\right) \approx \sin ^{2}\left(2 \theta_{13}\right) \sin ^{2}\left(\theta_{23}\right) \sin ^{2}\left(\frac{1.27 \Delta m_{32}^{2} L}{E}\right) \mp \frac{1.27 \Delta m_{21}^{2} L}{E} 8 J_{C P} \sin ^{2}\left(\frac{1.27 \Delta m_{32}^{2} L}{E}\right),
$$

where $J_{C P}$ is defined as:

$$
J_{C P}=\frac{1}{8} \cos \theta_{13} \sin \left(2 \theta_{12}\right) \sin \left(2 \theta_{23}\right) \sin \left(2 \theta_{13}\right) \sin \left(\delta_{\mathrm{CP}}\right) .
$$

Equation 1 shows the leading order CP-violating term but neglects the effect of matter on the oscillation probability. The sign of the second term is negative for neutrinos and positive for antineutrinos. Measuring the relative rate of electron neutrino appearance compared to electron antineutrino appearance gives sensitivity to $\sin \left(\delta_{\mathrm{CP}}\right)$ and therefore $\mathrm{CP}$ violation. Electron neutrino appearance also provides sensitivity to the octant of $\theta_{23}$ and the neutrino mass ordering. Discovering leptonic $\mathrm{CP}$ violation, measuring the $\theta_{23}$ octant and resolving the neutrino mass ordering are the three primary physics goals of the Hyper-K long-baseline programme.

\section{Hyper-K long-baseline event samples}

The Hyper-K neutrino oscillation sensitivities are estimated using the simulation of the T2K experiment [3]. The T2K far detector event rates are scaled to account for the larger Hyper-K 
detector and increased beam power, with a reduced scaling for events near the edge of the detector volume to account for the different ratio of surface area to volume for Hyper-K compared to SuperKamiokande. The studies presented here assume an exposure of $7 \times 10^{21}$ protons on target (POT) in neutrino beam mode and $20 \times 10^{21}$ POT in antineutrino beam mode. This exposure is equivalent to a 10-year running period, split 1:3 between neutrino and antineutrino beam modes.

The T2K event selection was applied to the scaled simulation to produce the Hyper-K event samples. The selection identifies events with a single Cherenkov ring that occur within the detector fiducial volume. The shape of the Cherenkov ring is used to distinguish muons, electrons and neutral pions, providing samples of single-ring muon-like and single-ring electron-like events. The reconstructed momentum of the muon candidate is required to be above $100 \mathrm{MeV} / \mathrm{c}$ to remove mis-reconstructed neutral current background events. In electron-like events the reconstructed neutrino energy, assuming charged-current quasi-elastic (CCQE) kinematics, is required to be below $1250 \mathrm{MeV}$, again to remove neutral current backgrounds. Finally, Michel electrons are tagged using delayed hits in the detector. Events are removed if they have more than one tagged Michel electron. The single-ring electron-like events are then classified into two samples, one with no Michel tag and one with a single Michel electron. In the case that $\sin \left(\delta_{\mathrm{CP}}\right)=0$, Hyper-K will select approximately $2300 v_{e}$ and $9300 v_{\mu}$ events in neutrino beam mode and $1900 \bar{v}_{e}$ and $12300 \bar{v}_{\mu}$ events in antineutrino beam mode. The event samples are shown in Fig. 1 while Fig. 2 shows the effect of varying $\delta_{\mathrm{CP}}$ on the electron-like samples.
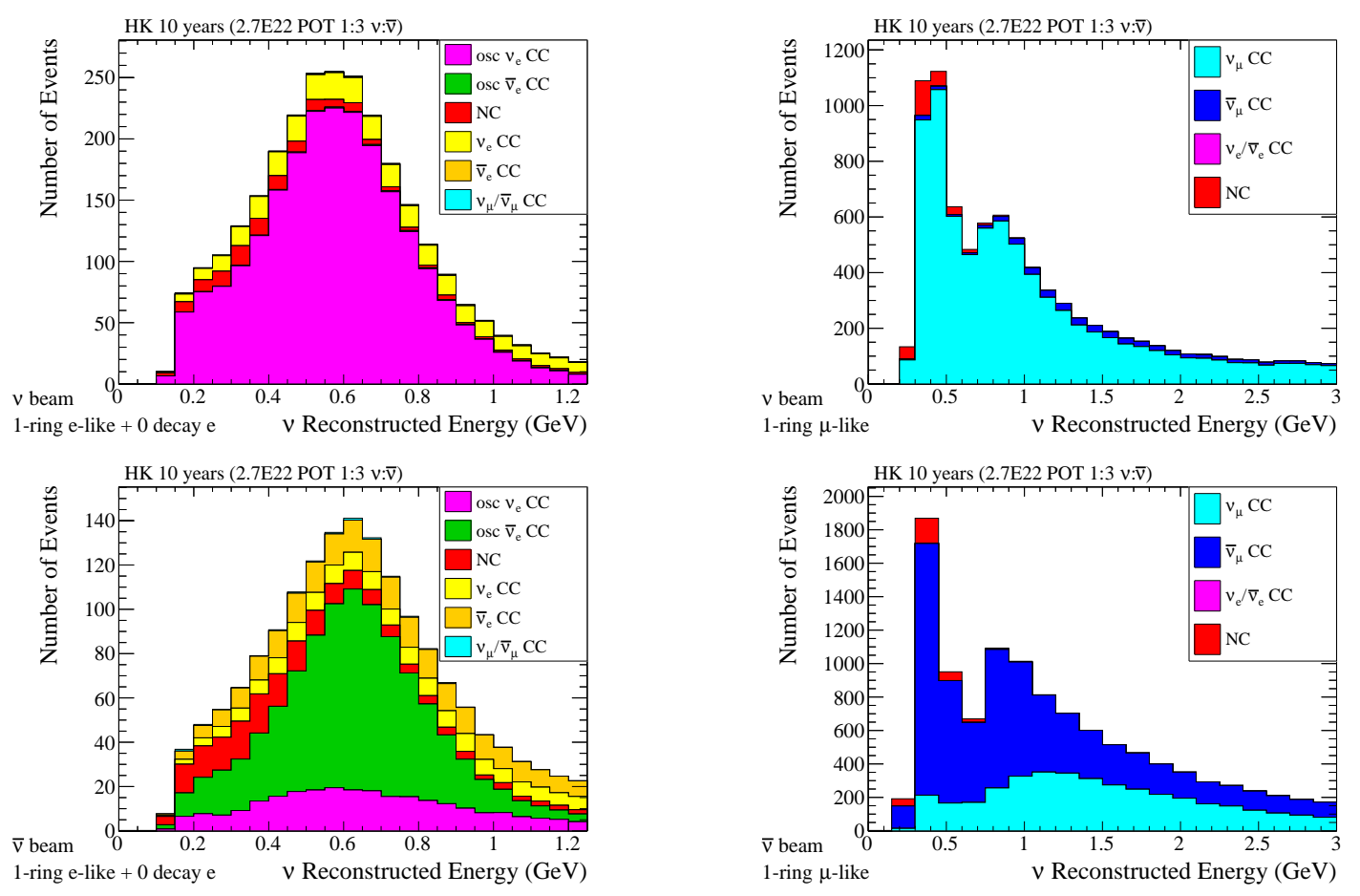

Figure 1: The Hyper-K event samples after 10 years of data taking assuming a 1:3 ratio between neutrino beam (top) and antineutrino beam (bottom). The single-ring electron-like samples are shown on the left and the single-ring muon-like samples are on the right, as a function of reconstructed neutrino energy assuming CCQE kinematics. 

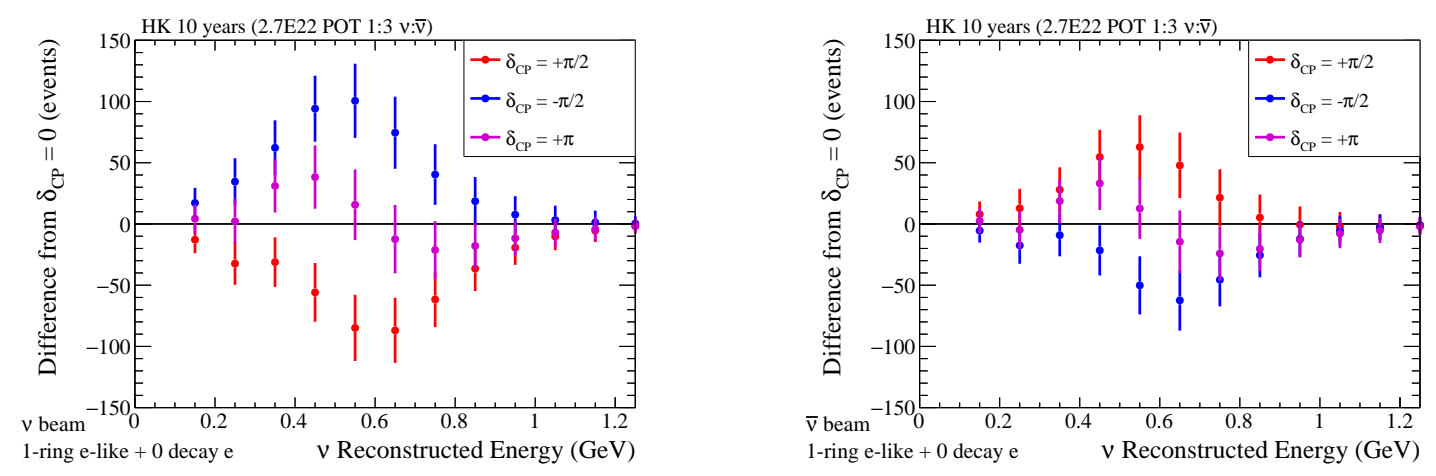

Figure 2: The difference in the Hyper-K single-ring electron-like event samples between different values of $\delta_{\mathrm{CP}}$ and the case for $\delta_{\mathrm{CP}}=0$. The samples assume 10 years of data taking with a 1:3 ratio between neutrino beam (left) and antineutrino beam (right). Statistical errors on the sample differences are shown.

\section{Hyper-K long-baseline sensitivity}

The Hyper-K long-baseline studies use the same analysis procedure as the T2K oscillation analyses [3]. Parameterised models of the neutrino flux and neutrino interaction cross-section are fit to simulated near detector data to provide a prediction of the unoscillated far detector event rate. The fit both tunes the models to the data and produces a smaller uncertainty on the far detector event rate. The tuned predicted event rate is then fit to the far detector event samples to extract the oscillation parameters.

For this analysis the T2K near detector fit results from Ref. [3] are used as an initial estimate of the model uncertainties after the near detector fit. A set of improved uncertainties is generated by scaling the T2K result according to the increase in statistics expected for the Hyper-K near detectors and taking into account the additional capabilities of the proposed new and upgraded Hyper-K near detectors. This scaling reduces the cross-section and flux parameter uncertainties by a factor of approximately 2.5 , with the limitation that no individual parameter error should be below $1 \%$. The initial uncertainty on the Hyper-K event samples using the T2K error model is shown in Tab. 1. The improved uncertainty is shown in Tab. 2.

\begin{tabular}{l|ll|lll} 
& \multicolumn{2}{|c|}{ 1-ring $\mu$-like } & \multicolumn{3}{c}{ 1-ring $e$-like } \\
Error source & $v$-mode & $\bar{v}$-mode & $v$-mode 0 d.e. & $\bar{v}$-mode 0 d.e. & $v$-mode 1 d.e. \\
\hline \hline Flux + Cross Section & $3.27 \%$ & $2.95 \%$ & $4.33 \%$ & $4.37 \%$ & $4.99 \%$ \\
Detector+FSI & $3.22 \%$ & $2.76 \%$ & $4.14 \%$ & $4.39 \%$ & $17.77 \%$ \\
\hline All syst & $4.63 \%$ & $4.10 \%$ & $5.97 \%$ & $6.25 \%$ & $18.49 \%$
\end{tabular}

Table 1: Percentage error on event rate by error source and sample, for the T2K-2018 syst error model. The Flux + Cross Section line takes into account the parameter correlations from the near detector fit.

The Hyper-K sensitivity studies were performed using the nominal oscillation parameter values shown in Tab. 3. Unless otherwise stated the fits do not include the atmospheric neutrino events and they assume that the neutrino mass ordering is known.

Figure 3 shows the significance with which CP conservation can be excluded as a function of the true value of $\delta_{\mathrm{CP}}$. For the improved systematics case, this shows that Hyper-K can discover CP 


\begin{tabular}{l|ll|lll} 
& \multicolumn{2}{|c|}{ 1-ring $\mu$-like } & \multicolumn{3}{c}{ 1-ring $e$-like } \\
Error source & $v$-mode & $\bar{v}$-mode & $v$-mode 0 d.e. & $\bar{v}$-mode 0 d.e. & $v$-mode 1 d.e. \\
\hline \hline Flux + Cross section & $0.81 \%$ & $0.72 \%$ & $2.07 \%$ & $1.88 \%$ & $2.21 \%$ \\
Detector+FSI & $1.68 \%$ & $1.58 \%$ & $1.54 \%$ & $1.72 \%$ & $5.21 \%$ \\
\hline All syst & $1.89 \%$ & $1.74 \%$ & $2.56 \%$ & $2.53 \%$ & $5.63 \%$
\end{tabular}

Table 2: Percentage error on event rate by error source and sample, for the Hyper-K improved error model.

\begin{tabular}{c|c} 
Parameter & Value \\
\hline $\sin ^{2} \theta_{23}$ & 0.528 \\
$\sin ^{2} \theta_{13}$ & $0.0218 \pm 0.0007$ \\
$\sin ^{2} \theta_{12}$ & 0.307 \\
$\left|\Delta m_{32}^{2}\right|(\mathrm{NH}) /\left|\Delta m_{13}^{2}\right|(\mathrm{IH})$ & $2.509 \times 10^{-3} \mathrm{eV}^{2} / \mathrm{c}^{4}$ \\
$\Delta m_{21}^{2}$ & $7.53 \times 10^{-5} \mathrm{eV}^{2} / \mathrm{c}^{4}$ \\
$\delta_{\mathrm{CP}}$ & $-\pi / 2$ \\
Mass Hierarchy & Normal
\end{tabular}

Table 3: Nominal values for oscillation parameters used in these studies.

violation at $5 \sigma$ significance for over $60 \%$ of the possible values of $\delta_{\mathrm{CP}}$ with an exposure of $27 \times 10^{21}$ POT. The difference between the statistics-only sensitivity and the two curves with systematics

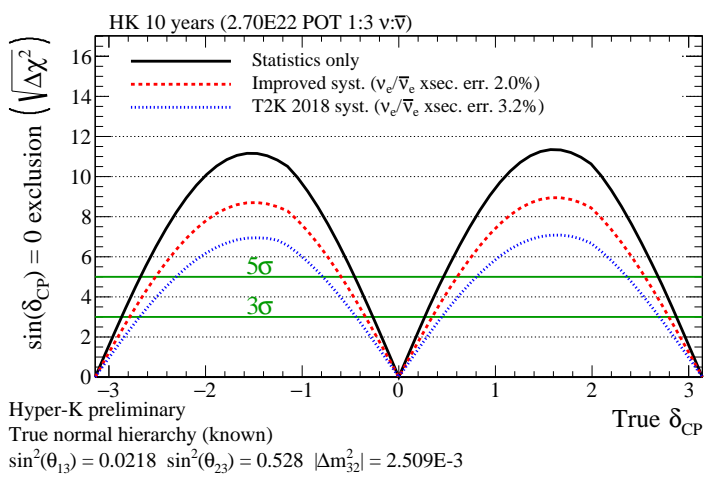

Figure 3: Significance with which Hyper-K can exclude $\mathrm{CP}$ conservation as a function of the true value of $\delta_{\mathrm{CP}}$. The black curve shows the result with statistical errors alone, dashed red includes the improved Hyper-K systematic errors and dotted blue is the T2K 2018 error model.

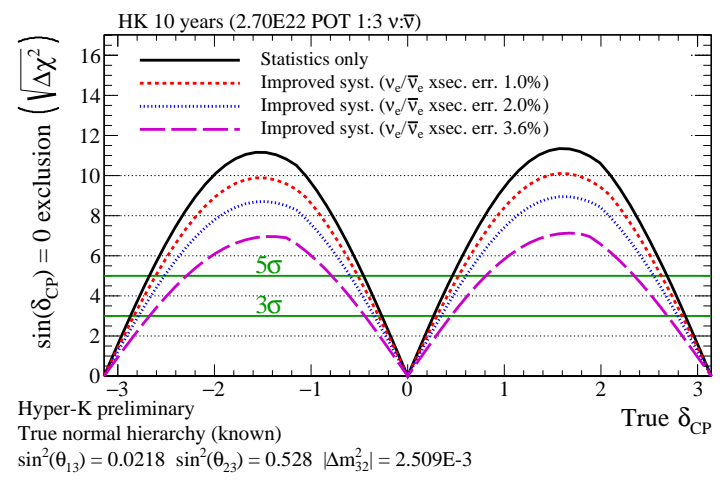

Figure 4: Significance to exclude CP conservation as a function of the true value of $\delta_{\mathrm{CP}}$, comparing different uncertainties on the $v_{e} / \bar{v}_{e}$ cross-section ratio. The black curve shows the result with statistical errors alone, dashed red indicates an uncertainty on the cross-section ratio of $1 \%$, dotted blue corresponds to a $2 \%$ uncertainty and the long-dash magenta corresponds to $3.6 \%$.

shows that this is a systematics limited measurement. This limitation is driven by the uncertainty in the $v_{e} / \bar{v}_{e}$ cross-section ratio, as shown in Fig. 4. As such, measuring the $v_{e} / \bar{v}_{e}$ cross-section ratio precisely is essential to achieve the Hyper-K long-baseline physics goals. The fraction of true values of $\delta_{\mathrm{CP}}$ for which $\mathrm{CP}$ violation can be discovered at a given significance is shown in Fig. 5 
as a function of exposure. This highlights that $\mathrm{CP}$ conservation can be excluded at $3 \sigma$ significance for $50 \%$ of the possible values of $\delta_{\mathrm{CP}}$ after two years of operation. Figure 6 shows the significance

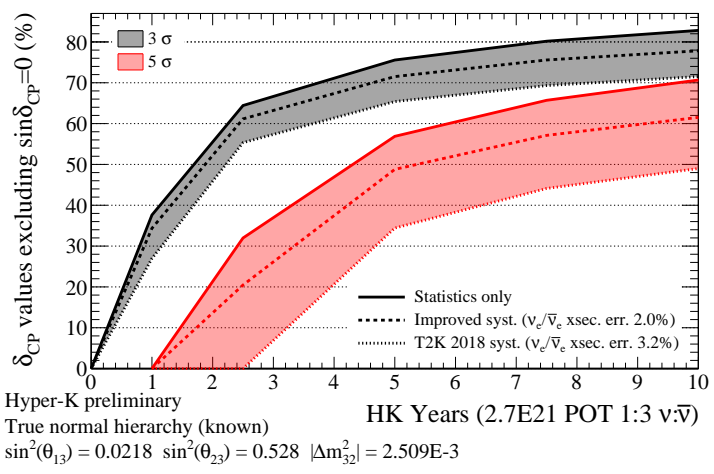

Figure 5: The fraction of true values of $\delta_{\mathrm{CP}}$ for which $\mathrm{CP}$ conservation can be excluded at $3 \sigma$ (black) and $5 \sigma$ (red) significance, as a function of exposure. The width of the bands shows how this changes for the statistics-only case, the improved Hyper-K error model and the T2K 2018 error model.

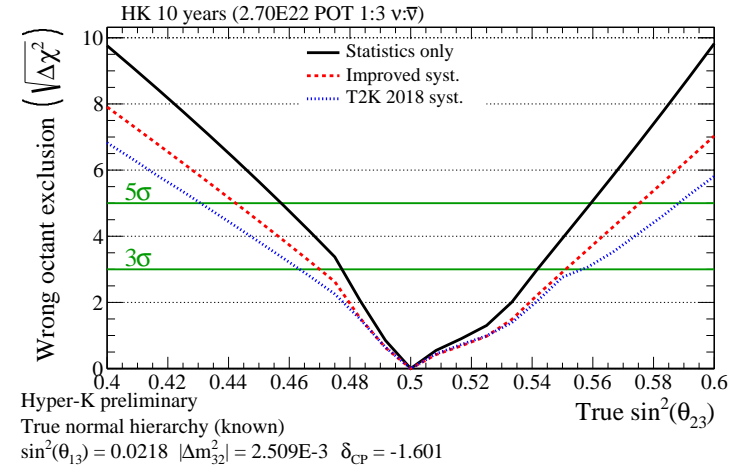

Figure 6: Significance with which the incorrect octant can be excluded as a function of the true value of $\sin ^{2} \theta_{23}$. The black curve shows the result with statistical errors alone, dashed red includes the improved Hyper-K systematic errors and dotted blue is the T2K 2018 error model.

with which the wrong octant of $\theta_{23}$ can be excluded, as a function of the true value of $\sin ^{2} \theta_{23}$. This shows the Hyper-K can determine the octant at $3 \sigma$ significance if the true value of $\sin ^{2} \theta_{23}$ is below 0.47 or greater than 0.55 .

\section{Conclusions}

The sensitivity of the Hyper-K long-baseline analysis to the neutrino oscillation parameters has been presented, including an updated systematic error model. The order of magnitude increase in the number of $v_{e}$ and $\bar{v}_{e}$ events compared to T2K will allow Hyper-K to discover CP violation at $5 \sigma$ significance for over $60 \%$ of the possible values of $\delta_{\mathrm{CP}}$ with an exposure of $27 \times 10^{21}$ POT. This will be a systematics limited measurement, and the dominant uncertainty comes from our lack of knowledge of the $v_{e} / \bar{v}_{e}$ cross-section ratio. Hyper-K will also be able to make precision measurements of other neutrino oscillation parameters, with sensitivity to both the $\theta_{23}$ octant and the neutrino mass ordering.

\section{References}

[1] A.D. Sakharov, Violation of CP Invariance, $C$ asymmetry, and baryon asymmetry of the universe, Pis'ma Zh. Eksp. Teor. Fiz. 5 (1967) 32.

[2] C. Hagedorn et al., CP Violation in the Lepton Sector and Implications for Leptogenesis, Int. J. Mod. Phys. A33 (2018) 1842006 [1711.02866].

[3] T2K collaboration, Search for CP Violation in Neutrino and Antineutrino Oscillations by the T2K Experiment with $2.2 \times 10^{21}$ Protons on Target, Phys. Rev. Lett. 121 (2018) 171802. 